\title{
Bocio Endémico en Pirque
}

\author{
Dr. Santiago Muzzo B. 1 ; Dr. Rodrigo Aguirre D.2; Dr. Alvaro Téllez T.2; \\ Dr. Sergio Valdivieso F. 2 ; T.M. Laura Leiva B.
}

\section{Endemic Goiter in a Chilean Rural Area}

\begin{abstract}
After four years operation of a National Program consisting in iodine diet supplementation through table salt a $30 \%$ prevalence of endemic goiter, mainly Grade I, was found among 223 children from a rural area placed at the hillsides of The Andes Range near Santiago, Chile. Symptoms and functional disturbances of thyroid gland showed no significant relationship with nutritional status or socio-economical conditions of the affected children. Studies aimed to detect goitrogenic substances on the population's normal diet as well as inadecuatc entichment of common salt with iodine are in their way in the country.
\end{abstract}

Se esti a que doscientos millones de personas en el mundu tie.sen bocin. La cifra ha experimen. tado escasas variaciones en las últimas décadas, a pesar que la mayoría de los países en que existe el problema han legislado para agregar yodo a la sal de mesa como medida profiláctica colectiva.

Desde 1885 investigaciones parciales en Chile, han demostrado la presencía de bocio endémico en varias áreas del país, especialmente en zonas cordilleranas'. En 1962 y 1972 se detectaron dos zonas de alta prevalencia en Pedregoso $(39,5 \%)$ y Pirque $(39,12 \%)$, respectivamente ${ }^{2-3}$.

Se cree que el déficit de yodo es la causa principal del bocio y del cretinismo endémico, pues al tratar con yodo las poblaciones afectadas, se puede disminuir drásticamente la prevalencia de ambos ${ }^{4}$. Sin embargo, existen otros factores etiológicos, entre ellos los bocígenos naturales de algunos alimentos (repollo, nabos, etc.) adèmás de otros presentes en tierras húmeđas. También se encuentra mayor prevalencia de bocio en poblaciones de nivel socioeconómico bajo, lo que estaría relacionado con mayor tasa de desnutrjción y contaminación bacteriana. Además, se ha descrito que la frecuencia de tiroiditis es mayor en zona de bocio endérnico ${ }^{5 \cdot 6 \cdot 7-14}$.

Se sabe que la desnutrición postnatal precoz produce un retraso del crecimiento irrecuperable que deja como secuela una menor talla y circunferencia craneana final ${ }^{8}$.

Se ha descrito que poblaciones de nivel socioeconómico bajo tienen alta prevalencia de des. nutrición y mayores tasas de retardo mental que las de nivel socioeconónico medio $y$ alto. Ein este retraso mental influyen la desnutrición precoz, la

\footnotetext{
1 Instituto de Nutrición y Tecnología de los Alimentos, Unidad de Endocrinología, Universidad de Chile.

2 Departamento de Saludi. Comuna de Pirque.
}

Trabajo financiado en parte por un Grant de la Junta Nacional de Auxilio Escolar y Becas. menor estimulación sicosensorial que reciben estos niños, además de déficit específicos de nutrientes, entre ellos el yodo?.

En Chile hay conciencia del bocio endémico y se legisló sobre la yodación de la sal de mesa en 1958, sin embargo, la legislación sólo se aplicó desde 1978 .

Este estudio se diseñó con el propósito de conocer la prevalencia del bocio endémico en los escolares de Pirque (área de alta prevalencia), 4 años después de jniciar la yodación de la sal de mesa, analizar sus características y relaciones con el nivel socioeconómico, estado nutricional y talla actual.

\section{MATERIAL Y METODO}

Durante el curso del año 1982 se tomó una muestra aleatoria de 223 niños (13\% del total) 118 mujeres y 105 hombres, representativa y proporcional de los 1.712 nifios que asisten a las escuelas de educación básica de la comuna de Pirque.

En ellos se estudió la prevalencia y las características del bocio. Se efectuó un examen médico para pesquisar signos, síntomas o ambos, de hipotiroidismo, se interrogó sobre antecedentes mórbidos del encuestado y sus familiares directos. Se midieron peso y talla con balanza de precisión y cartabón vertical respectivamente.

Los bocios se clasificaron en Grado I (Tíroides palpable por lo menos del tamaño de la última falange del pulgar del encuestado, visible sólo en retroflexión cervical); JI (Tiroides visible en posición cervical normal, mayor tamaño que el descrito para Grado I); III (visible en posición cervical normal, desde considerable distancia, con importante aumento de tamaño a la palpación), y 0 (personas que no cumplen con ninguna de las características anteriores).

El estado de nutrición se clasificó mediante las tablas de peso para la talla según edad y sexo, del 
National Center of Health Statistics (OMS). Se diagnosticó desnutrición cuando el peso para la talla era menor del $90 \%$, Eutrofia entre 90 y $110 \%$, Sobrepeso entre 110 y $120 \%$ y Obesidad sobre los $120 \%$ del pesa para la talla.

La estatura se calificó de acuerdo con las Tablas de talla para edad y sexo, del National Center of Health Statistics (OMS) 13. Se consideró que habia retraso estatural si la talla para la edad era igual o inferior al $95 \%$, talla adecuada entre 95 y $105 \%$ y exceso de talla sobre $105 \%$.

El nivel socioeconómico (NSE) se definió mediante la escala de Graffar Modificada, que toma en cuenta la escolaridad y ocupación del Jefe de Hogar, características de la vivienda (calidad, propiedad, abastecimiento de agua, disposición de excretas y equipamiento del hogar), $y$ permite estratificar la población en 6 niveles ${ }^{10}$.

El significado estadístico de los resultados se evaluó por medio de $\mathrm{X}^{2}$.

\section{RESULTADOS}

La Tabla 1 muestra la prevalencia de bocio encontrada en los escolares de Pirque, que alcar. zó a 30\%. Del total de los bocios, el grado I constituyó un $86,6 \%$, el grado II, $11.9 \%$ y el III, $1,5 \%$. A la palpación de la glándula tiroídea tuvo una consistencia normal en $92 \%$ de los casos y sólo en $8 \%$ se consideró aumentada. Los signos y síntomas de alteración de función tiroídea estudiados evaluando pulso arterial, piel, pelo, irrita. bilidad, frecuencia de deposiciones $y$ actividad física, demostraron escasa incidencia y sin diferencias entre los escolares con y sin bocio.

Tabla 1.

Prevalencia de bocio en escolares de Pirque

\begin{tabular}{|c|c|c|c|}
\hline & & $\mathrm{N}$ & $\%$ \\
\hline \multirow{4}{*}{ Bocio } & 0 & 156 & 70,0 \\
\hline & I & 58 & 26.0 \\
\hline & II & 8 & 36 \\
\hline & [II & 1 & 0,4 \\
\hline & & 223 & 100,0 \\
\hline
\end{tabular}

En la Tabla 2 se aprecia que no habian escolares en los dos niveles más al tos de Graffar, sólo $0,6 \%$ pertenecía al nivel $3,35,5 \%$ al nivel 4 , $63,3 \%$ en nivel 5 y sólo $0,6 \%$ al nivel 6 de miseria. No se encontró relación significativa entre bocio y estrato socioeconómico.

La Tabla 3 señala que de los escolares de sexo femenino $14,4 \%$ tenían obesidad, $25,4 \%$ sobre- peso y sólo $9,3 \%$ desnutrición. El estudio estadístico no demostrá relación entre bocio y estado nutricional. Entre los escolares de sexo masculino habian $7,8 \%$ con obesidad, $23,3 \%$ con sobrepeso y $6,8 \%$ con destrutrición, sin relación estadísticamente significativa entre bocio y esta. do nutritivo. (Tabla 4).

Tabla 2.

Nivel socioeconómico de los escolares de Pirque (Graffar Modificado)

\begin{tabular}{|c|c|c|c|c|c|c|}
\hline Nivel & \multicolumn{2}{|c|}{ Con Bocia } & \multicolumn{2}{|c|}{ Sin Bocic } & \multicolumn{2}{|c|}{ Total } \\
\hline $\begin{array}{l}3 \\
4 \\
5 \\
6\end{array}$ & $\begin{array}{c}20 \\
44 \\
-\end{array}$ & $\begin{array}{l}- \\
31.3 \\
68.7\end{array}$ & $\begin{array}{r}1 \\
56 \\
91 \\
1\end{array}$ & $\begin{array}{r}0.6 \\
37.6 \\
61.2 \\
0.6\end{array}$ & $\begin{array}{r}1 \\
76 \\
135 \\
1\end{array}$ & $\begin{array}{r}0.6 \\
35.5 \\
63.3 \\
0.6\end{array}$ \\
\hline Total & 64 & 100.0 & 149 & 100.0 & 213 & 1000 \\
\hline
\end{tabular}

Tabla 3.

Estado nutricional de escolares de sexo femenino de Pirque

\begin{tabular}{|c|c|c|c|c|c|c|}
\hline$W_{0}$ peso talla & $\mathbf{N}$ & bocic & $\mathrm{N}^{2}$ & Bocio & $\cdot \mathrm{N}$ & lal ${ }_{\alpha}$ \\
\hline $\begin{array}{ll}<90 & \\
90-110 \\
110 & 120 \\
>120 & \end{array}$ & $\begin{array}{r}7 \\
39 \\
22 \\
10\end{array}$ & $\begin{array}{r}9.0 \\
20.0 \\
28.0 \\
13.0\end{array}$ & $\begin{array}{r}4 \\
21 \\
8 \\
7\end{array}$ & $\begin{array}{l}10.0 \\
52.5 \\
20.0 \\
1.5 .5\end{array}$ & $\begin{array}{l}11 \\
60 \\
30 \\
17\end{array}$ & $\begin{array}{r}9.3 \\
50.9 \\
25.4 \\
14.4\end{array}$ \\
\hline Tu:al & $7 \beta$ & 100.0 & 40 & 100.0 & 118 & 100.8 \\
\hline
\end{tabular}

Tabla 4.

Estado nutricional en escolates de sexo masculino de Pjrque

\begin{tabular}{|c|c|c|c|c|c|c|}
\hline$\%$ Fesoltalla & $\lambda$ & So: io & N & $\underset{\%}{B 0 c i o}$ & $N$ & tal \\
\hline $\begin{array}{l}<90 \\
90 \cdot 110 \\
110 \quad 120 \\
>120\end{array}$ & $\begin{array}{r}6 \\
48 \\
16 \\
6\end{array}$ & $\begin{array}{r}8.0 \\
630 \\
210 \\
80\end{array}$ & $\begin{array}{r}1 \\
16 \\
8 \\
2\end{array}$ & $\begin{array}{r}3.7 \\
59.7 \\
29.6 \\
7.4\end{array}$ & $\begin{array}{r}7 \\
64 \\
24 \\
8\end{array}$ & $\begin{array}{r}6.8 \\
63.1 \\
23.3 \\
7.8\end{array}$ \\
\hline Total & 76 & 100 & 27 & 100.0 & 103 & 100.0 \\
\hline
\end{tabular}

La Tabla 5 muestra que $44,9 \%$ de las niñas tenía adecuación de talla para la edad menor de $95 \%$, el resto de la población estuvo sobre esta adecuación. No se encontró relación entre la presencia de bocio y la calidad de estatura. En los varones $45,7 \%$ presentó adecuación estatural menor de1 $95 \%$, siendo el resto de estatura normal. Nuevamente no hubo relación estadistica entre bocio y caliclad de estatura. (Tabla 6). 
Tabla 5

Calidad de estatura en escolares de sexo femenino de Pirque

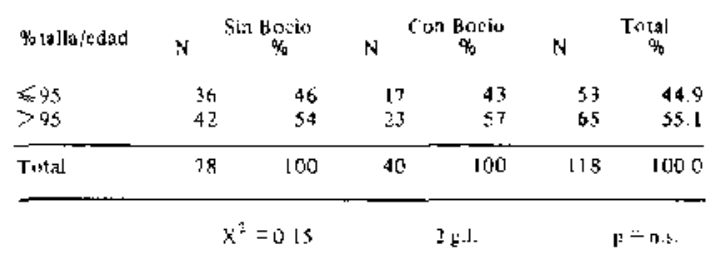

Tabla 6 .

Calidad de estatura en escolares de sexo masculino de Pirque

\begin{tabular}{lrrrrrr} 
\% talla/edad & Nirt Bocjo & \multicolumn{2}{c}{ Cun Bucio } & \multicolumn{2}{c}{ Total } \\
$\leqslant y 5$ & 36 & 46 & 12 & 44 & 48 & 45.7 \\
395 & 42 & 54 & 15 & 56 & 57 & 54.3 \\
\hline Total & 78 & 100 & 27 & 100 & 10.5 & 100.0
\end{tabular}

$\mathrm{x}^{2}=0.00 \mathrm{~s}$

2 .. .

$\mathrm{F}=$ n.s.

\section{DISCUSION}

Los resultados obtenidos en este trabajo indjcan que la prevalencia de bocio en los escolares de Pirque, aún permanece alta, siendo levemente inferior a los porcentajes demostrados por Domínguez y cols. en 1974. La mayor parte de estos bocios fue Grado I.

Ha sido descrito que al administrar yodo a las poblaciones que presentan bocio endémico pueden tardar algunos años en bajar la prevalencia, pero porcentualmente el bocio grado I se hace más frecuente, disminuyendo los grados II y III 11

El hecho de no encontrar signos de alteración de función tiroidea, hace sospechar que los escolares con bocio son eutiroídeos, hecho que se demostró mediante determinaciones de $\mathrm{TSH}, \mathrm{T}_{3}$ y $\mathbf{T}_{\mathbf{4}}$ plasmáticos (datos por publicar).

Esta población de escolares corresponde a un nivel socioeconómico (NSE) medio-bajo, perteneciendo el $99 \%$ a los niveles 4 y 5 con gran homogeneidad en los casos estudiados, lo que explicaría la falta de relación entre presencia de bocio y NSE, relación que ha sido demostrada en otros trabajos con NSE heterogéneo, donde se explicaría que existiera más bocio a menor NSE por la mayor frecuencia de desnutrición, contaminación ambiental y menor ingesta de yodos.

Tanto los escolares de sexo femenino como masculino presentan importantes porcentajes de déficit de estatura, que podrian ser explicados por factores genéticos, o desnutrición en la infancia.
En esta población de escolares hemos encontrado mayores porcentajes de consanguinidad que en la población general 7,2 y $2,4 \%$ respectivamente (datos por publicar). Sin embargo, no encontramos diferencias en los porcentajes de consanguinidad entre los escolares con y sin bocio. Este hecho indicaría que los factores genéticos no serían la principal causa del bocio.

Dado el nivel socioeconómico de esta población es posible que estos escolares hubiesen tenido problemas nutricionales en su primera infancia, to que podria haber causado por lo menos en parte el retraso estatural que presentan, sin embargo la estatura similar de los escolares con y sin bocio indica que de haber existido este problema habría sido influido en igual forma en ambos grupos.

El estado nutritivo demostró alta prevalencia de obesidad y sobrepeso que concuerda con las tasas que hemos encontrado en población de igual nivel socioeconómico de Región Metropolitana (por publicar) siendo mayores en la mujer que en el varón y con muy bajo porcentaje de desnutrición.

La falta de diferencia entre los estados nutritivos de ambos grupos estudiados estaria indicando que el estado nutricional actual no jugaría un rol en la presencia de bocio en el grupo estudiado.

Estos resuitados indican que el bocio endémico es aún prevalente en los escolares de un área de alta endemia en Chile. Este hecho no seria secundario a un problema de malnutrición actual o pasada por déficit global de nutrientes de estos escolares. Sin embargo, factores tales como inadecuada yodación de la sal, factores genéticos, bocigenos naturales y mayor incidencia de tiroiditis en esta población escolar ${ }^{14}$ sugieren la necesidad de continuar estudiando estos escolares y efectuar una adecuada vigilancia de yodación de la sal y de la existencia de bocígenos en alimentos y agua 12

En próximos trabajos, se mostrarán los hábitos de consumo de distintos alimentos que podrian estar influyendo en este problema y los estudios de laboratorio de función tiroidea de estos escolares.

\section{RESUMEN}

Se estudió la prevalencia de bocio en los escolares del área de Pirque, cuatro años después de iniciada la yodación de sal en Chile. Se encontró un $30 \%$ de bocio principalmente de grado I. No se encontraron síntomas de alteración de la función tiroídea. No hubo relación entre bocio con nivel socioeconómico, estado 
nutritivo y talla actual. Los resultados indican que Pirque aún es zona de alta endemia de bocio, sin tener relación con estado nutricional y nivel socioeconómico. Se discuten los factores causales de bocio endémico.

\section{AGRADECIMIENTOS}

Nuestros agradecimientos a las señoras: Irene Trufello y Teresa Segure, de la Unidad de Estadistica $y$ Computación del Lnstituto de Nutrición y Tecnología de los Alimentos por su apoyo estadístico y a la señorita Viola Lyon por su trabajo secretarial.

\section{REFERENCIAS}

1 Romero, H.: El bocio como problema de salubridad. Rev. Chilena Hig. Méd. Prev, 5: 423, 1943.

2 Nogel, R., Etcheverry, R., Guzmán, C., Hille, A., Barzelatto, J., Covarrubias. E.: Encuesta de bocio endémico en la población mapuche y en algunos colegios de primera enseñanza de las provincias de Cautin y Malleco. Rev. Med. Chile 90: 616, 1962.

${ }^{3}$ Dominguez, M., Quesney, F., Michaud, P., Maggiolo. $C$. Ugarte, $J .:$ La prevalencia del bocio en escolares de la comuna de Puente Alto, Pirque y San José de Maipo. Estudio clínico epidemiológico. Rev. Med. Chile. 102: 639, 1974,

4 Phoruah, P.O.D., Buttfield, I.H., Hetzel, B.S.; Neurological damage to the fetus resulting from severe iodine deficiency during pregnancy. The Lancet I: $308,1971$.

5 Vought, R.L., Brown, F.A., Sibinovic, K.H.: Anti- thyroid compound(s) produced by Escherichia coli: Preliminary reports. J. Clin. Endocr. 38: 861, 1974.

${ }^{6}$ Schnitzer, M., Kahm, S.V.: Humic substances in the environment. New York, Marcel Dekker, 1972.

7 Madeiros-Neto, G.: General nutrition and endemic goiter. In Fndemic Goiter and Endemic Cretinism. Stanbury, J., Hetzel, B. (eds). Wiley Medical publication. p. 269,1980 .

8 Monckeberg, F.: La desnutrición en el niño y sus consecuentias. Rev. Cent. Lstudios Educ. (México) $3: 67,1973$.

9 Fierro Benitez, R., Ramiez, I., Estrella, E, and Stonbury, $\boldsymbol{J}$.B.: The role of iodine in intellectual development in an area of endemic goiter. In: Endemic Goiter and Cretinism: Continuing Threats of World Health, Dunn, J.T., Madeijos Neto, G. (eds). Report of the IV Meeting of the PAHO Technical group on Endemic Goiter. PAHO Sci. Publ. NO 292, p. 135,1974 .

10 Alvarez, M.L.. Wurgaft, F., Salazar, M.E.: Mediciones de nivel socioeconómico bajo urbano en tamilias con lactante desnutrido. Arch. Latinoamer. de Nutr. 32 (3): 650,1982 .

11 Perinetti, H.: Ll bocio endémico en la provincia de Mendoza y su cradicación por la sal enriquecida con yodo. Bol. Acad. Nac. Med. (Bs. Aires) 47: 319, 1969.

12 Michaud, P., Silva, J.E.: Contenido de yodo en una muestra de sal yodada. Rey. Med. Chile 109: 681, 1981.

13 NHCS: Growth curves for children birth-18 years. United States - Vital and lealth Statistics. Data from the National Health Survey. Serie Il. Number 165.

14 Michaud, P., Téllez, R., Aguirre, R., Téllez, A. y Valdivieso, $S_{.1}$ Anticuerpos antitiroideos y prueba de yodo-perclorato de potasio en escolares con y sir bocio endémico. Rey. Med. Chile 111: 1029, 1983. 